\title{
Biologia reprodutiva de Elaenia chiriquensis (Lawrence) (Aves, Tyrannidae) em Cerrado do Brasil Central
}

\author{
Rita de C. S. de Medeiros \& Miguel Â. Marini
}

Programa de Pós-Graduação em Biologia Animal, Instituto de Ciências Biológicas, Universidade de Brasília. 70910-900 Brasília, Distrito Federal, Brasil.

\begin{abstract}
Reprodutive biology of Elaenia chiriquensis (Lawrence) (Aves, Tyrannidae) in the Cerrado of the Brazil Central. Elaenia chiriquensis (Lawrence, 1865) is a very common passerine abundant during the reproductive season (September-December) in the cerrados of Central Brazil. Since its breeding biology is still poorly known, this study had the aim to describe several aspects of its reproduction. We conducted the study at the "Estação Ecológica de Águas Emendadas", Federal District, Brazil, between August 2002 and December 2003. Birds were banded $(\mathrm{n}=285)$ during this period. Nests $(\mathrm{n}=110)$ found were monitored every 1-5 days. Clutch sizes had one $(n=14)$, two $(n=88)$ or three eggs $(n=1)$, which were pinkish with small reddish dots around the obtuse end. Nest characteristics were comparable to other records of this species. Breeding occurred between mid September to the end of December with two peaks of active nests, one in mid October and one in mid November. These two peaks may be explained by a second breeding attempt after a successful nest or a second breeding attempt after an unsuccessful first attempt. Thirty-two nests (30\%) were successful, with most of the nests being depredated $(n=70 \%)$ and few abandoned $(n=4 \%)$. Egg success was of $28 \%$ and the hatching rate was $0.96 \pm 0.02$. Fledgling production was of 0.48 fledglings/nest and fecundity was 1.1 fledglings/female. All these values are similar to the ones already reported for this species and similar to other Neotropical passerines.
\end{abstract}

KEY WORDS. Clutch size; fecundity; nest; reproductive success.

RESUMO. Elaenia chiriquensis (Lawrence, 1865) é um Passeriforme abundante nos cerrados do Planalto Central entre os meses de setembro e dezembro, quando se reproduz. Este trabalho tem por objetivo descrever alguns aspectos de sua biologia reprodutiva. O estudo foi desenvolvido na área da Estação Ecológica de Águas Emendadas, Planaltina, Distrito Federal durante o período de agosto de 2002 a dezembro de 2003. Ninhos $(n=110)$ foram monitorados a cada 1-5 dias. Adultos $(\mathrm{n}=285)$ foram individualmente marcados. $\mathrm{O}$ tamanho das ninhadas variou entre um $(n=14)$, dois $(n=88)$ ou três ovos $(n=1)$. Elaenia chiriquensis constrói ninhos abertos em forma de "taça", com ovos de coloração branco gelo a rosado e pequenas manchas de cor ferrugínea no pólo obtuso. Dimensões e forma dos ninhos foram comparáveis aos da mesma espécie na América do Sul. A reprodução ocorreu entre meados de setembro a fins de dezembro, com dois picos de ninhos ativos, meados de outubro e novembro. Estes dois picos podem ser explicados por uma segunda tentativa após o sucesso ou insucesso da primeira nidificação. Trinta e dois ninhos (30\%) obtiveram sucesso, 70 foram predados (67\%) e 4 abandonados (4\%). O sucesso dos ovos foi de $28 \%$ e a taxa de eclosão de $0,96 \pm 0,02$. A produção anual de filhotes $(0,48$ filhotes/ninho), a taxa de fecundidade ( 1,1 filhotes/fêmea), bem como as demais taxas encontradas foram semelhantes para outros estudos sobre a espécie e para outros Passeriformes neotropicais em países da América do Sul.

PALAVRAS-CHAVE. Fecundidade; ninho; sucesso reprodutivo; tamanho de ninhada.

Reprodução é um processo biológico fundamental na história de vida dos seres vivos, requerendo um suprimento de energia adicional onde os indivíduos possuem tempo e recursos limitados à sua disposição. Além disso, a reprodução inclui riscos e representa um investimento que geralmente afeta adversamente a vida do reprodutor (RICKLEFs 1990). As aves variam em muitos aspectos de suas histórias de vida (RICKLEFs 2000), principalmente tratando-se de sua reprodução. Indivíduos em determinada idade e sexo, durante o período reprodutivo defrontam-se com uma série de escolhas (como por exemplo, defender ou abandonar o ninho diante de algumas situações), as quais determinam seu padrão reprodutivo (WILLIAMs 1966). Elaenia chiriquensis albivertex (Pelzeln, 1868) éum Passeriforme pertencente à família Tyrannidae cujas espécies caracte- 
rizam-se por possuírem pequeno tamanho, padrão discreto de coloração e pouco dimorfismo sexual (RIDGELY \& TUDOR 1994). A distribuição da espécie estende-se desde a Costa Rica até Missiones na Argentina e somente não é encontrada na Caatinga, porção centro-oeste do sul da Amazônia, Santa Catarina, Rio Grande do Sul, Chilee Uruguai (PInto 1944, SchAuenseE 1982, SICK 1997). É migratória na região do Brasil Central (NEGRET et al. 1984, Marini \& Cavalcanti 1990, Sick 1997) sendo um tiranídeo bastante comum no Cerrado do Planalto Central entre os meses de agosto e dezembro quando chega para reproduzir. Representa a ave mais capturada em redes de neblina durante o período chuvoso (ALVES 1990). As rotas migratórias e datas desses deslocamentos ainda não se encontram bem esclarecidas devido entre outros fatores, a coexistência de populações migratórias e residentes (MARINI \& CAVALCANTI 1990).

O grande número de indivíduos observados é retratado ainda na quantidade de ninhos presentes entre os meses de setembro e dezembro. Em razão da abundância de indivíduos de E. chiriquensis no Cerrado relacionado a seu hábito alimentar predominantementefrugívoro, acreditamos tratar-se de uma espécie chave no que diz respeito à dispersão de sementes de plantas do Cerrado. Portanto, a escolha de E. chiriquensis como tema deste trabal ho deveu-se a abundância marcante da espé cie, importância biológica e escasso conhecimento de sua biologia reprodutiva, resumindo-se a informações históricas, qualitativas e pontuais (Partridge 1964, Belton 1985, Stiles \& SkUtch 1989). Tal carência de informações básicas acerca da biologia reprodutiva da maioria das espécies comuns do Cerrado é um dos fatores que dificultam propostas de manejo e conservação. Assim, o objetivo deste estudo é descrever alguns aspectos da biologia reprodutiva da espécie, tais como: período reprodutivo, descrição de ninhos e ovos com seus padrões morfométricos, tempo de incubação, taxa de fecundidade das fêmeas e tempo de permanência dos filhotes nos ninhos, comparando-os com outras espécies neotropicais.

\section{MATERIAL E MÉTODOS}

\section{Área de estudo}

O trabalho foi realizado no período de agosto de 2002 a dezembro de 2003 na Estação Ecológica de Águas Emendadas (ESECAE), Unidade de Conservação localizada a 50 km de Brasília,

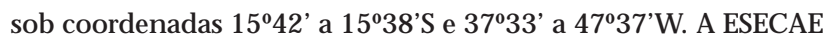
contempla um fragmento de 10.500 ha de Cerrado do Brasil Central composto por várias fitofisionomias (SILVA JR. \& FELFILI 1996). Foi delimitada uma área de 100 ha $(1 \times 1$ km) com marcação de 400 quadrados de 50 × 50 m, utilizando-se GPS (Global Positioning System) do tipo GARM IN, bússola e trenas. Esta área se localiza na porção sudeste da ESECAE e é composta por um mosaico de fitofisionomias do Cerrado contendo porções de cerrado típico, campo sujo, campo limpo e parque cerrado. Maiores detalhes sobre a vegetação podem ser encontrados em SiLvA JR. \& FELFILI (1996) e sobrea fauna em MarinHo-FILHo et al. (1998).

O Cerrado é considerado o segundo maior bioma do Bra- sil e da América do Sul (Ribeiro \& W Whter 1998) cobrindo uma extensão aproximada de 1,8 milhões de quilômetros quadrados (AB'SABER 1977, 1983, Silva 1998) e considerada a maior e mais ameaçada savana tropical do mundo (SILVA \& BATES 2002). O efeito sazonal no Cerrado é marcadamente relevante, constituído por duas estações claramente definidas: a estação seca efria (maio a setembro) e outra quente e chuvosa (outubro a abril) (EITEN 1993), sendo o clima classificado como tropical chuvoso (Aw de Köpen) (EITEN 1984). A precipitação média na região do Distrito Federal varia entre 1500 e $1750 \mathrm{~mm}$ com temperaturas entre 20 a 26C (NIMER 1979). Dados referentes à precipitação mensal na área da Estação Ecológica de Águas Emendadas foram obtidos junto ao Instituto Nacional de Meteorologia (INMET) provenientes da Estação Meteorológica de Brasília.

A região do entorno da ESECAE é constituída por propriedades particulares predominando chácaras, sítios e fazendas. MACHADO et al. (1997) observaram nas proximidades da Unidade de Conservação, a expansão da malha viária com o conseqüente crescimento das áreas urbanas em direção aos limites da Estação. Tal incremento na urbanização local está gerando um aumento do processo de insularização da Unidade.

O gênero Elaenia possui 17 espécies registradas até o momento e caracteriza-se por alto grau de similaridade morfológica entre algumas delas. Possuidoras de rica vocalização, a dificuldade na identificação em campo tem sido resolvida através das vocal izações típicas de cada espécie, demonstrando dessa forma que sua comunicação éantes acústica que visual (CAMARGO 1986, SICK 1997).

Na ESECAE, o gênero encontra-se representado por seis espécies: E. flavogaster (Thunberg, 1822), E. spectabilis (Pelzeln, 1868), E. parvirostris (Pelzeln, 1868), E. mesoleuca (Deppe, 1830), E. cristata (Pelzeln, 1868) e E. chiriquensis, sendo esta última registrada em ambiente de mata de galeria, cerrado típico e campo sujo (BAGNo 1988). Indivíduos adultos de E. chiriquensis apresentam crista pequena com variável quantidade de branco não aparente, garganta parda tingindo-se em cor verde-oliváceo no peito eamarelo embranquecido no ventre, com bordas das asas amarelo claro e regiões dorsais cor verde-oliváceo. Possui duas barras opacas na porção terminal das asas e metatarso escuro (NICEFORO \& Olivares 1976, Stiles \& Skutch 1989, Ridgely \& Tudor 1994).

\section{Anilhamento}

$\mathrm{O}$ anilhamento dos adultos foi realizado entre agosto de 2002 e julho de 2003. Utilizaram-se redes de neblina ( $12 \mathrm{~m}$ de comprimento por 2,5 $\mathrm{m}$ de altura com malha de $36 \mathrm{~mm}$ ), distribuídas em linhas e colunas dentro da área delimitada e permanecendo no mesmo local durante dois dias não consecutivos. O objetivo da captura dos indivíduos em redes de neblina foi averiguar a existência de placas de incubação e a presença de jovens. As capturas foram realizadas entre as 6:00 e 12:00 h ou até ocorrer queda muito acentuada da atividade das aves. Como as fêmeas são as únicas responsáveis pela construção do ninho e incubação dos ovos (SKUTCH 1966, WetMORE 1972), apenas os indivíduos capturados com placa de incubação pude-

Revista Brasileira de Zoologia 24 (1): 12-20, março 2007 
ram ser sexados. A extensão do período reprodutivo não permitiu a identificação segura do sexo daqueles que não apresentavam placas de incubação, identificados dessa forma como sexo indeterminado.

Estimou-se o status jovem ou adulto dos indivíduos capturados através do grau de ossificação do crânio, presença/ausência de comissura labial e estado de conservação da plumagem. As aves capturadas receberam uma anilha metálica (fornecidas pelo CEMAVE/IBAMA) no metatarso direito e uma combinação individual de três anilhas coloridas no metatarso esquerdo.

\section{Procura e monitoramento de ninhos}

A procura por ninhos foi realizada em árvores e arbustos dentro da área demarcada, entre outubro e dezembro de 2002 e entre agosto e dezembro de 2003. Alguns ninhos foram encontrados através da observação de casais de E. chiriquensis apresentando aparente comportamento sexual de corte, cantos em dueto ou acompanhando indivíduos transportando material de construção de ninhos ou alimento para filhotes.

Assim que um ninho era localizado, a identificação da espécie era realizada através de observação à distância aproximada de 20 m, mediante a vocalização do indivíduo adulto. Após encontrar o ninho, estes eram marcados à distância mínima de $5 \mathrm{~m}$ e numerados com fitas plásticas pelo qual eram posteriormente identificados. Ninhos foram monitorados através de visitas com intervalos de dois a cinco dias onde seu status (ativo ou não ativo) era registrado, ovos e filhotes quantificados, pesados e medidos quando necessário. Durante fases críticas dos ninhos tais como postura, eclosão e partida dos filhotes, monitorou-se os ninhos a interval os de até um dia visando maior acuidade nas estimativas. As visitas eram tão breves quanto possíveis no intuito de minimizar impactos relacionados à presença humana. $\mathrm{O}$ monitoramento dos ninhos com altura acima de dois metros era realizado com ajuda de um pequeno espelho amarrado a uma haste, permitindo assim diminuir o impacto das visitas.

Os ninhos cuja espécie foi identificada e que apresentaram no mínimo um ovo foram registrados como válidos. Considerou-se como bem sucedidos os ninhos em que pelo menos um filhote voou. Monitoraram-se todos os ninhos encontrados em 2002 e 2003 até que se tornassem inativos. Consideraram-se como ninhos predados aqueles encontrados vazios e intactos, total ou parcialmente destruídos no chão ou no próprio local, ou quando ovos destruídos (ou danificados) e filhotes mortos por meio de danos físicos fossem encontrados. $\mathrm{Ni}$ nhos vazios quando filhotes ainda não haviam alcançado a idade mínima que permitissem deixar o ninho, também foram considerados predados. Admitiu-se como abandonados como sendo aqueles em que os ovos permaneciam no ninho sem eclodir por mais de 15 dias ou filhotes mortos no ninho intacto aparentando estado de inanição.

\section{Características dos ninhos e ovos}

Foi dada preferência à realização das medidas do ninho nos estágios iniciais de incubação evitando a deformação natural decorrente de seu uso. A morfometria dos ninhos foi rea- lizada através das medidas, em milímetros, de diâmetro externo, diâmetro interno, altura do ninho e profundidade da câmara oológica. Esta última medida corresponde ao ponto central da câmara até a borda do ninho. A altura em relação ao solo $(\mathrm{cm})$ foi medida de sua borda ao chão com auxílio de trena comum. As medidas do ninho foram realizadas com o auxílio de paquímetro com precisão de 0,05 mm. Foram ainda medidas as distâncias entre ninhos ativos simultâneos e próximos $(<30 \mathrm{~m})$ visando à obtenção da distância média entre os mesmos e de uma estimativa de sua densidade.

Devido à sua fragilidade, os ovos foram pesados e medidos (comprimento e largura) uma única vez, evitando sempre que possível sua mani pulação nos dois primeiros dias do período de incubação. Observou-se ainda seu aspecto morfológico (cor e forma), além do número de ovos por ninho. Devido à grande quantidade de ovos encontrada, optamos por medir e pesar apenas pequena parte deles $(n=27)$ com auxílio de paquímetro com precisão de 0,05 $\mathrm{mm}$ e dinamômetro de $10 \mathrm{~g}$ com precisão de $0,1 \mathrm{~g}$. O volume dos ovos foi calculado através da equação de Hort (1979): Volume $=0,51 \times$ (comprimento) $\mathrm{x}$

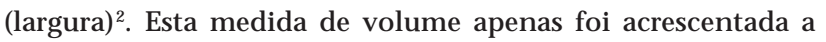
título de proporcionar um maior número de parâmetros ao estabelecido neste trabalho.

\section{Cálculo de taxas}

O período de incubação foi definido como sendo aquele compreendido entre a postura do segundo ovo e eclosão do primeiro, não sendo considerado o período de postura. O período de crescimento dos ninhegos foi definido como sendo aquele entre a eclosão do primeiro ovo e saída do último filhote do ninho (Robinson et al. 2000). Para análise dos dados referentes ao período de incubação, consideraram-se apenas aqueles ninhos encontrados antes da postura do primeiro ovo e que foram monitorados até a eclosão. Realizaram-se cál culos simples de porcentagem de predação, abandono e sucesso dos ninhos.

A taxa de eclosão dos ovos (M AYFIELD 1975) foi calculada dividindo-se o número de filhotes nascidos pelo número de ovos existentes antes da eclosão. Calculou-se a taxa de eclosão para cada um dos ninhos em separado, encontrando a média \pm EP. Foram descartados dessa análise ninhos predados ainda na fase de incubação sem estabilização da quantidade de ovos, bem como ninhos encontrados já com filhotes.

Calculou-se a taxa de fecundidade anual das fêmeas como o número de filhotes que abandonaram osninhos dividido pelo número de fêmeas (Aguilar et al. 1999, SAether \& BaKke 2000) e que representa o número de filhotes eclodidos de todas as ninhadas encontradas em 2003 dividido pelo número de fêmeas presentes. Este último valor refere-se ao número de ninhos encontrados (uma fêmea por ninho) e não às fêmeas capturadas em redes, uma vez que podem existir fêmeas não reproduzindo na área de estudo ou ainda fêmeas sem placas de incubação capturadas e que desenvolveram placa a posteriori.

A porcentagem do sucesso dos ovos (SKUTCH 1966) é calculada pela seguinte razão ( $\mathrm{n}$ o de filhotes que voaram $/ \mathrm{n}$ ㅇ de

Revista Brasileira de Zoologia 24 (1): 12-20, março 2007 
ovos colocados), multiplicados por 100. Para o cálculo da taxa de produção anual de filhotes, RickLefs \& BLoom (1977) sugerem tratar-se da razão entre o número total de filhotes que voaram e o número total de ninhadas, o que foi realizado em relação aos ninhos de 2003.

\section{RESULTADOS}

Foram monitorados na área de estudo 110 ninhos, sendo 10 ninhos oriundos de estudos preliminares realizados na estação reprodutiva de 2002 e 100 ninhos em 2003.

\section{Período reprodutivo}

Em 2003, as aves iniciaram a construção dos ninhos na segunda quinzena de setembro quando passamos a observar adultos transportando material para construção e o último ninho acompanhado tornou-se inativo no dia 26 de dezembro. Observou-se uma intensidade anormal de chuva para o mês de agosto $(62,4 \mathrm{~mm})$ com um inesperado decréscimo da precipitação em setembro (11,3 mm) evidenciando que o ano de 2003 foi um ano atípico quando comparado com a precipitação média mensal para o período de 1990-2000 (Fig. 1). A data da migração da espécie está evidentemente relacionada a seu período reprodutivo, mostrando que apesar da chuva perdurar até maio, a reprodução pode se estender no máximo a início de janeiro. Existem registros de capturas de jovens ainda na área até o mês de fevereiro.

Foram capturados 285 indivíduos (sem distinção entre sexos) de E. chiriquensis na área da grade na ESECAE, sendo 145 indivíduos em 2002 e 140 em 2003. A observação dos primeiros indivíduos presentesna área, data da segunda quinzena deagosto. Nos meses de setembro a dezembro de 2002 e dezembro de 2003 capturou-se 75 indivíduos apresentando placa de incubação coincidindo com os registros de ninhos ativos. Foram capturados 30 indivíduos com mudas de dorso, corpo e asa entre outubro de 2002 e fevereiro de 2003, o que provavelmente indica a presença de indivíduos jovens, além de adultos realizando mudas pós-reprodutivas. Dentre estes indivíduos, em janeiro e fevereiro de 2003, 10 deles apresentavam crânio não ossificado e presença de plumagem nova, tratando-se provavel mente de indivíduos jovens que ainda permaneciam na área (Fig. 2). A quantidade de ovos atingiu dois picos durante a estação reprodutiva, na primeira dezena de outubro e segunda dezena de novembro. A quantidade de ninhos ativos com filhotes também ocorreu em dois picos, primeiro entre 10 e 20 de outubro e o segundo entre 10 e 20 de novembro de 2003 (Fig. 3).

\section{Ninhos}

Os 110 ninhos encontrados de E. chiriquensis possuíam o formato de taça ou tigela rasa apoiado pelo fundo em bifurcação ( $n=106)$ ou trifurcação $(n=4)$ de gal hos e sua coloração é amarronzada em decorrência do material utilizado. O corpo do ninho propriamente dito é composto por uma intrincada rede de fibras vegetais frouxas dispostas em forma circular. Dentre estas fibras podemos destacar uma grande quantidade

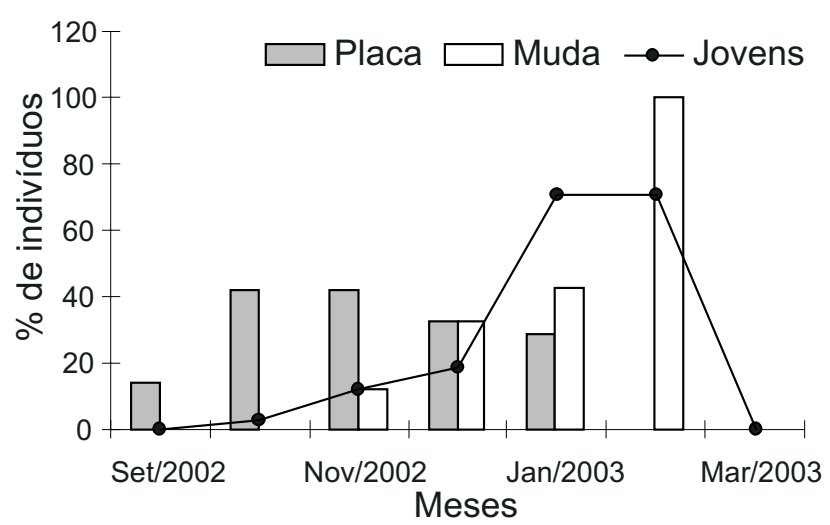

Figura 1. Porcentagem de indivíduos adultos capturados com placa de incubação, mudas e jovens ao longo dos meses compreendidos entre setembro de 2002 e março de 2003 na ESECAE/DF.

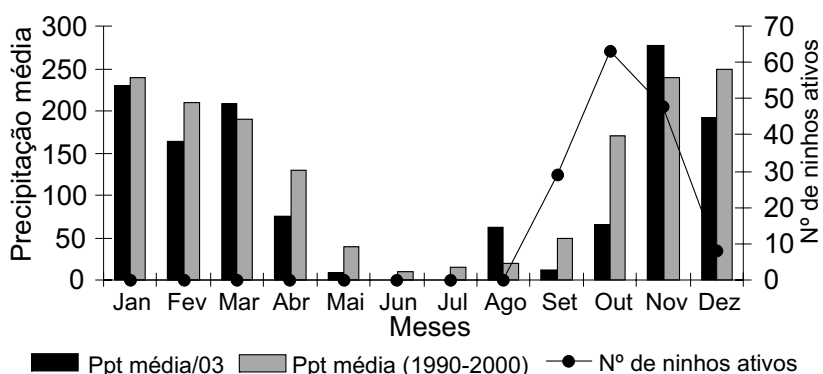

Figura 2. Precipitação média $(\mathrm{mm})$ registrada pela Estação Climatológica de Brasília/INMET durante 1990, 2000 e 2003 para a ESECAE e número de ninhos ativos encontrados.

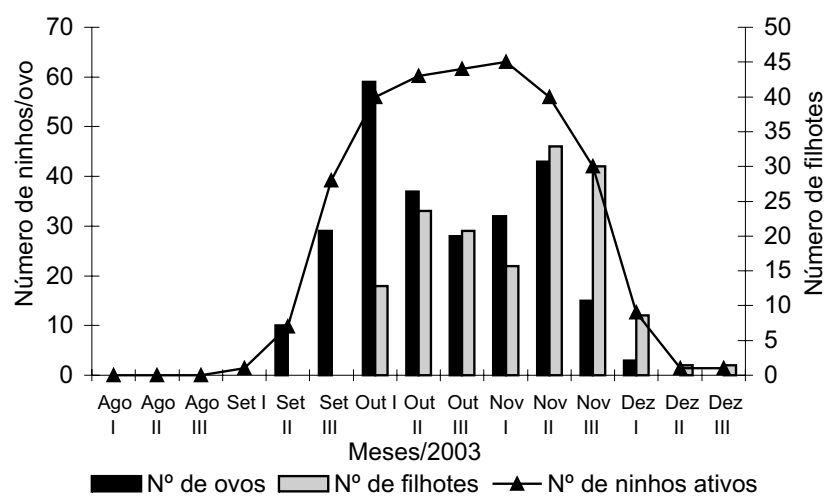

Figura 3. Ninhos ativos durante os meses correspondentes à estação reprodutiva de 2003 com quantidades de ovos e filhotes. Os meses foram divididos em períodos de 10 dias (I, II, III).

de inflorescências de gramíneas em sua maioria sem sementes, onde com maior expressividade, foram encontradas àquelas pertencentes ao gênero Paspalum (Graminea). O período médio

Revista Brasileira de Zoologia 24 (1): 12-20, março 2007 
Tabela I. Características morfológicas dos ninhos de E. chiriquensis com alturas em relação ao solo e distância entre ninhos.

\begin{tabular}{lcccc}
\hline \multicolumn{1}{c}{ Variáveis } & $\mathrm{n}$ & Média & E.P. & CV(\%) \\
\hline Diâmetro externo (mm) & 27 & 63,7 & 1,0 & 8 \\
Diâmetro interno (mm) & 27 & 46,5 & 0,6 & 7 \\
Altura do ninho (mm) & 27 & 33,0 & 1,2 & 19 \\
Profundidade (mm) & 27 & 25,6 & 0,7 & 14 \\
Altura do ninho em relação ao solo (m) & 108 & 1,6 & 0,3 \\
Distância entre ninhos consecutivos em uma & 11 & 27,7 & 1,8 & 21 \\
mesma estação reprodutiva (m) & & & \\
\hline
\end{tabular}

de construção do ninho é de 5 dias $(n=10)$ variando de 4 a 7 dias. Realizou-se a morfometria de 27 ninhos, bem como medidas de altura em relação ao solo de 108 ninhos (Tab. I).

Ovos

Os ovos têm forma ovóide de cor creme claro podendo variar entre branco gelo a branco rosado contendo pequenas manchas de diferentes tamanhos variando da cor ferrugem a marrom escuro, concentradas ao redor do polo obtuso. A massa média dos ovos $(n=30)$ foi de $1,8 \pm 0,04 \mathrm{~g}(\mathrm{CV}=14 \%)$. 0 comprimento médio e a largura média $(n=30)$ encontrada foram de 18,2 $\pm 0,2 \mathrm{~mm}(\mathrm{CV}=6 \%)$ e $13,9 \pm 0,2 \mathrm{~mm}(\mathrm{CV}=7 \%)$ respectivamente. O volume médio $(\mathrm{n}=30)$ calculado segundo a fórmula de Hort (1979) foi de 1789,4 $\pm 41,8 \mathrm{~mm}^{3}$ (CV =13\%).

As ninhadas foram de um $(n=14)$, dois $(n=88)$ e três $(n=1)$ ovos. Não puderam ser levadas em consideração para esta análise as posturas pertencentes a ninhos predados antes que o segundo ovo pudesse ser posto. De um total de $103 \mathrm{ni}-$ nhos, $85 \%$ continham dois ovos ou dois filhotes pelo menos em um dos monitoramentos realizados.

Taxas de eclosão, mortalidade, fecundidade e produção anual de filhotes

Durante as duas estações reprodutivas foram monitorados 110 ninhos, dos quais $63 \%(n=70)$ foram predados, $4 \%(n=4)$ abandonados e $33 \%(n=36)$ bem sucedidos. Não foram observadas perdas de ninhos ocasionadas pela intensidade das chuvas. A taxa de fecundidade ( $\mathrm{n}$ - de filhotes eclodidos $\mathrm{n}=118 / \mathrm{n}$ 응 de fêmeas reproduzindo, $n=110$ ) foi de 1,1 filhotes/fêmea. $A$ taxa média de eclosão ( $\mathrm{n}$ o de filhotes eclodidos / $\mathrm{n}$ ㅇ de ovos da ninhada) para 2002/2003 é igual a 1,0 $\pm 0,02(n=55)$. O tempo médio referente ao período de incubação $(n=17)$ foi de $13,4 \pm$ 1,5 dias ( $C V=11 \%$ ). A porcentagem do sucesso dos ovos foi de $28 \%$, calculada por meio do $\mathrm{n}$ - de filhotes que voaram, $n=53$ / no de ovos colocados, $\mathrm{n}=189 \times 100$, e a taxa de produção de filhotes (número de filhotes que voaram de todas as ninhadas do ano de 2003, $n=53$ / número de ninhadas de 2003, $n=110$ ) de 0,48 filhotes/ninhada.

A média calculada do ciclo do ninho, considerado entre a postura do primeiro ovo e a saída do filhote foi de 29,9 dias \pm 1,3 dias ( $n=31$ ). O tempo médio de construção (cinco dias) acrescido do intervalo entre a construção e a postura, que é muito variável, como já dito anteriormente, sugere que o período entre a construção do ninho e abandono deste pelos filhotes pode levar em torno de 37 a 40 dias.

\section{DISCUSSÃO}

As dificuldades que o gênero Elaenia oferecem quanto à sua diferenciação morfológica entre algumas espécies são amplamente conhecidas, principalmente devido a distribuição simpátrica de muitas delas, dentre elas E. chiriquensis (NAUMBURG 1930, Partridge 1964, Traylor JR 1982). Dentre as seis espécies identificadas para a ESECAE, quatro delas são raras em nossa área de estudo (E. flavogaster, E.spectabilis, E. mesoleuca e E. parvirostris), sendo preferencialmente encontradas em outros ambientes tais como nas bordas e interior das matas de galerias, veredas e campo sujo. As demais, E. chiriquensis e E. cristata, encontram-se predominantemente em cerrado sensu stricto e são facilmente distinguíveis em campo pela altura da crista, porte, comportamento e vocalização.

O período de nidificação de $\mathrm{E}$. chiriquensis para os quais se registrou ninhos ativos, entre setembro e dezembro, assemelhase ao encontrado para a espécie na Argentina (PARTRIDGE 1964) e para suas congêneres no Brasil (BeLton 1985). BeLton (1976), em seu trabalho sobre aves do Rio Grande do Sul, registra gônadas aumentadas para espécies do gênero Elaenia entre os meses de setembro e janeiro. Várias espécies de Passeriformes da região centro-sul do país (Alves \& Cavalcanti 1990, Marini 1992) e outrostiranídeos (VAsconcelos \& Lombardi 1996, Aguilar et al. 1999) apresentam também registro de ninhos ativos coincidindo com a época de início das chuvas, sujeito este período a pequenas mudanças devido as variações geográficas e climáticas anuais.

A maior quantidade de ninhos ativos ocorreu no mês de novembro, entretanto isto representa a ocorrência de dois picos de nidificação, outubro e novembro, separados por aproximadamente 30 dias. O primeiro pico representa o início do período reprodutivo e o segundo pico pode ser explicado por pelo menos duas hipóteses: a) segunda tentativa de reprodução após o insucesso da primeira nidificação do ano; b) segunda reprodução após sucesso na produção da primeira ninhada do ano. Considerando que a maioria dos ninhos foi predado, a primeira hipótese deve explicar a maioria das ocorrências do

Revista Brasileira de Zoologia 24 (1): 12-20, março 2007 
segundo pico de nidificação. Entretanto, a segunda hipótese não pode ser totalmente descartada, pois foi possível constatar um caso de dupla nidificação pela mesma fêmea no mesmo período reprodutivo. Um ninho que obteve sucesso em 23/X/2003 foi reutilizado, com nova postura aproximadamente cinco dias depois da saída dos filhotes da primeira ninhada. Na mesma área, LOPES \& M ARINI (2005) trabalhando com Suiriri affinis (Burmeister, 1856) e S. islerorum (Zimmer, Whittaker \& Oren, 2001) detectaram para ambas as espécies, múltiplas tentativas de reprodução para a mesma estação reprodutiva, após sucesso e insucesso da nidificação. Segundo RoPER (2005), a habilidade das aves em voltar a nidificar influencia o processo reprodutivo, o que se torna mais importante para as aves tropicais que possuem períodos reprodutivos mais longos, permitindo a ocorrência de várias tentativas.

O pico de ninhos ativos ocorreu no mês de outubro e a maior precipitação foi registrada em novembro $(276,4 \mathrm{~mm})$ quando também se consideraram as chuvas mais fortes do segundo semestre de 2003 (dados obtidos no INMET). Além das chuvas, outras variáveis ambientais tais como temperatura, clima, umidade relativa do ar ou fotoperíodo provavelmente podem estar influenciando a determinação do período reprodutivo. Relações entre o período de nidificação e estação chuvosa tem sido freqüentemente relatadas para aves neotropicais (Aguilar et al. 2000, MEZquida 2002). Espera-se que as aves regulem seu ciclo reprodutivo com o regime das chuvas, quando os pais possuem maior quantidade de alimento disponível para alimentar seus filhotes (PerRins 1970). A relação entre período chuvoso e abundância de artrópodes nos trópicos foi considerada por diversos autores (KARR 1976) decorrendo que aves insetívoras sejam favorecidas pelo aumento na quantidade de artrópodes aéreos (ONIKI \& WILLIS 1983).

De maneira geral, os ninhos de E. chiriquensis assemeIham-se em aspecto com os descritos para as demais espécies do gênero (Traylor Jr 1982, de la Peña 1987, Chatellenaz \& FERRARO 2000), bem como para outros representantes da família (PICHORım et al. 1996). A construção do ninho sobre forquilhas de dois a três gal hos também parece ser um padrão encontrado para o gênero (I HeRING 1900, WetMoRe 1972, FFrENCH 1991). Das oito espécies de tiranídeos estudadas por Mezquida (2002), sete delas possuem ninhos do tipo aberto com formato de taça ou tigela com exceção feita a Myarchus tyrannulus (Muller, 1776) que nidifica normalmente em ocos de árvores.

O período médio de construção do ninho foi semelhante ao encontrado por MezQuidA (2002) para Griseotyrannus aurantiotrocristatus (d'Orbigny \& Lafresnaye, 1837) na Argentina. Acreditamos ser bastante comum variações em períodos de construção de ninhos, uma vez que muitos fatores podem estar envolvidos na escolha do local de nidificação e estrutura do ninho (MARTIN 1993). Ninhos que possuem um acabamento mais consistente podem levar mais tempo para serem concluídos e estar relacionados à experiência da fêmea em nidificar.

Períodos curtos de incubação e crescimento de filhotes são benéficos por diminuir o tempo de exposição do ninho à predação em ambos os períodos (MARTIN 1987). O período de incubação (13 dias) de E. chiriquensis na área de estudo foi um pouco menor que aquele encontrado para a espécie no sudeste da Costa Rica, 14 dias (SKUTCH 1945). Acreditamos que a pequena diferença encontrada deve-se à metodologia adotada por Skutch, que determinou como sendo o período de incubação aquele compreendido entre a postura do segundo ovo e a eclosão do mesmo. Para E. flavogaster, FFrENCH (1991) detectou que o período de incubação vai de 15 a 17 dias em Trindade e Tobago. São comuns pequenas variações temporais relacionadas ao comportamento reprodutivo das aves que dependem das condições ambientais e da disponibilidade de alimento (Murphy 1986).

O tamanho da ninhada de E. chiriquensis assemelha-se ao encontrado para a espécie em outros países da América do Sul (SKutch 1945, Wetmore 1972, Ffrench 1991) evidenciando que o mais comum para a espécie é a postura de dois ovos, embora possa ocorrer a postura de um ovo em menor proporção e raramente três, conforme encontrado também para outras espécies do gênero (SKUTCH 1985). A postura média de dois ovos é comum quando se trata de tiranídeos como o observado por Mezquida (2002) em seis das oito espécies estudadas.

A produção de ovos representa um processo oneroso relacionado às demandas alimentares, de forma que o suplemento alimentar pode afetar o tamanho da ninhada, a duração da postura e o intervalo entre as posturas (MonAGHAM \& NAGER 1997). O limite na quantidade de ovos postos parece estar mais relacionado à capacidade dos pais de cuidarem da prole do que propriamenteà energia necessária para colocar mais ovos (MARTIN 1987). Com exceção de poucas espécies, os tiranídeos apresentam um tamanho de ninhada bastante uniforme, em número de dois (SKUTCH 1985). Dentre os Passeriformes relatou-se um número de ninhada mais variável sendo que relativamente pouca pesquisa tem sido desenvolvida quanto ao efeito da filogenia no tamanho da ninhada neste grupo (Yom-Tov et al. 1994). Espécies iguais localizadas em latitudes diferentes apresentam o mesmo tamanho de ninhada (Yom-Tov et al. 1994), o que de fato foi possível constatar no caso de E. chiriquensis.

No colorido e na forma, os ovos de E. chiriquensis confirmam a descrição realizada por BLAKE (1956) e assemelham-se aos de Elaenia albiceps (Orbigny \& Lafresnave 1837) e Elaenia obscura (Orbigny \& Lafresnave 1837) (INHERING 1900). O intervalo de tempo encontrado entre a postura dos ovos (1 a 2 dias) é o mesmo descrito por Ffrench (1991). Para os tiranídeos temse observado que o ritmo da postura é um ovo a cada dois dias (SKUTCH 1960). Em oito espécies de tiranídeos registrou-se para sete delas um ritmo de postura em dias alternados e variação regional no intervalo de postura, exceção feita à $T$. savana que apresentou um ritmo diário (MEzQuida 2002). Estas diferenças podem estar relacionadas à disponibilidade de alimento, acarretando provavelmente efeitos sobre a produtividade anual de filhotes (Mezquida 2002).

Revista Brasileira de Zoologia 24 (1): 12-20, março 2007 
O tempo médio de permanência dos filhotes no ninho (15 dias) foi idêntico ao encontrado por SKUTCH (1945) para a espécie. O período mais vulnerável na vida de aves nidícolas ocorre em seu desenvolvimento inicial, na fase de ninhegos, quando se encontram confinados ao ninho e totalmente dependentes dos pais (LIEBEZEIT \& GEORGE 2002). O crescimento de filhotes nas diferentes famílias de aves é atribuído à abundância de recursos alimentares (GEBHARDT-HENRICH \& RICHNER 1998). O estágio de crescimento dos filhotes exige consideráveis demandas de energia, que por sua vez é determinado pelo padrão de eclosão, taxa de crescimento, tamanho da ninhada e seus custos regulatórios, cada um destes podendo influenciar a limitação de alimento do filhote (MARTIN 1987).

A taxa de sucesso dos ovos (28\%) foi superior a encontrada por SKUTCH (1966) em estudo realizado na Costa Rica (22\%). O sucesso dos ovos de espécies neotropicais possui valores mais baixos que as taxas de Passeriformes da América do Norte que chegam a atingir $80 \%$ de sucesso (Icterus spurius) (Linnaeus, 1766) (Icteridae) (Rıcklefs \& BLoom 1977). Variações de taxas entre localidades geográficas são comuns e dados originários de amostras simples não permitem comparação estatística uma vez que a aquisição de dados ocorre através de metodologias distintas (RıckLeFs \& BLOOM 1977).

A taxa de eclosão dos ovos de E. chiriquensis na área de estudo $(1,0)$ foi bastante elevada quando comparada à encontrada $(0,35)$ por SKUTCH $(1966)$ em compilação de dados para aves neotropicais na Costa Rica. A eclosão dos ovos está associada à constância no processo de incubação, ou seja, interrupções freqüentes no aquecimento dos ovos por parte dos adultos acarreta um resfriamento dos mesmos comprometendo desta forma o desenvolvimento dos embriões (YERKEs 1998). Na área de estudo, poucos ovos de E. chiriquensis não eclodiram significando provavelmente uma constância de incubação elevada.

A fecundidade representa uma das taxas de difícil mensuração (COOKE \& Rockwell 1998). Segundo GILL (1989), a fecundidade anual precisa considerar o comprimento da estação reprodutiva e o número de filhotes sobreviventes após perdas como predação e abandono. Resolveu-se definir a taxa de fecundidade anual de fêmeas como sendo a razão entre o número de filhotes eclodidos de todas as ninhadas de 2003 e o número de fêmeas presentes, conceito este também adotado por Aguillar et al. (1999) e SAethe \& BaKke (2000) e que se acredita traduzir mais claramente a medida anual de capacidade fisiológica e individual de cada fêmea da espécie em deixar descendentes. Existe uma relação intrínseca e ainda não muito clara entre a fecundidade, idade da primeira reprodução, sobrevivência juvenil e longevidade (LINDÉN \& MøLLER 1989). Espécies migratórias sofrem custos reprodutivos maiores que espécies residentes. Atividades como migração, estabelecimentos de territórios para reprodução e mudas propiciam um gasto de energia extra que afeta a sobrevivência e a fecundidade dos adultos. Altas taxas de fecundidade parecem estar relacionadas à baixa sobrevivência de adultos, à baixa sobrevivência de juvenis ou ambos.
Elaenia chiriquensis apresenta suas taxas reprodutivas (eclosão, produção anual de filhotes, fecundidade das fêmeas e sucesso dos ovos) dentro dos padrões conhecidos para a espécie em outros países da América do Sul e outras espécies pertencentes à família Tyrannidae. A história de vida desta espécie aparentemente mostra um processo de seleção natural no sentido a favorecer a alta sobrevivência dos adultos uma vez que os altos índices de predação evidenciam um baixo sucesso reprodutivo.

\section{AGRADECIMENTOS}

A Jader Marinho-Filho, Regina Macedo, James J. Roper, Thais Aguilar, Charles Duca, Leonardo Lopes, Letice Câmara França e Mieko Kanegae. Apoio: CNPq.

\section{REFERÊNCIAS BIBLIOGRÁFICAS}

AB'SABer, A.N. 1977. Os domínios morfoclimáticos da América do Sul. Primeira aproximação. Geomorfologia 52:1-21.

AB'SABER, A.N. 1983. O domínio dos cerrados: introdução ao conhecimento. Revista do Servidor Público 11: 41-55.

Aguilar, T.M.; L.O. Leite \& M.Â. Marinı. 1999. Biologia da nidificação de Lathrotriccus euleri (Cabanis, 1968) (Tyrannidae) em fragmentos de mata em Minas Gerais. Ararajuba 7: 125133.

Aguilar, T. M.; M.M. Coelho \& M.Â. Marinı. 2000. Nesting Biology of the Gray-hooded Flycatcher (Mionectes rufiventris). Ornitologia Neotropical 11: 223-230.

Alves, M.A.S. 1990. Social system and helping behaviour in the white-banded Tanager (Neothraupis fasciata). Condor 92: 470-475

Alves, M.A.S. \& R.B. Cavalcantı. 1990. Ninhos, ovos e crescimento de filhotes de Neothraupis fasciata. Ararajuba 1: 91-94.

Bagno, M.A. 1988. As aves da Estação Ecológica de Águas Emendadas, p. 22-33. In: J. Marinho-Filho; F. Rodrigues \& M. GuIMARÃES (Eds). Vertebrados da Estação Ecológica de Águas Emendadas. Brasília, SEMATEC, IEMA, IBAMA, 92p.

BeLton, W. 1976. Taxonomy of certain species of birds from Rio Grande do Sul, Brazil. National Geographic Society Research Reports 15: 183-188.

Belton, W. 1985. Birds of Rio Grande do Sul, Brazil. Parte II. Formicaridae through Corvidae. Bulletin American Museum of Natural History 180: 1-241.

BLAKE, E.R. 1956. A colletion of panamanian nests and eggs. Condor 58: 386-388.

CAmargo, H.F.A. 1986. Contribuição ao estudo das espécies brasileiras do Gênero Elaenia (Aves: Tyrannidae). Boletim Centro de Estudos Ornitológicos 2: 6-19.

Chatellenaz, M.L. \& L.I. Ferraro. 2000. Materiales vegetales y fúngicos utilizados por aves em la construción de nidos en la noreste Argentino y Paraguay. Comunicaciones Científicas y Tecnológicas Corrientes 1: 1-4.

COOKE, F. \& R.F. RockweLl. 1998. Reproductive success in a lesser snow goose population, p. 237-243. In: T.H. Clutton-Brock 
(Ed.). Reproductive success. Studies of individual variation in contrasting breeding systems. Chicago, The University of Chicago Press, 575p.

DE LA PEÑA, M.R. 1987. Nidos y huevos de las aves argentinas. Santa Fé, Martin R. de la Peña, 263p.

Eiten, G. 1984. Vegetation of Brasília. Phytocoenologia 12: $271-$ 292.

EITEN, G. 1993. Vegetação do Cerrado, p. 17-73. In: M.N. Pinto (Ed.). Cerrado: caracterização, ocupação e perspectivas. Brasília, Universidade de Brasília, 657p.

FFrenCH, R. 1991. A guide to the birds of Trinidad and Tobago. Ithaca, Comstock Publishing Associates, 426p.

Gebhardt-HenRich, S. \& H. Richner. 1998. Causes of growth variation and its consequences for fitness, p. 441. In: J. M. StARCK \& R. RickLEFs (Eds). Avian growth and development. New York, Oxford University Press, 441p.

GILL, F.B. 1989. Ornithology. New York, W.H. Freeman \& Company, 660p.

HoY, D.F. 1979. Practical methods of estimating volume and fresh weight of bird eggs. Auk 96: 73-77.

I HeRING, H.V. 1900. Catálogo crítico-comparativo dos ninhos e ovos das aves do Brasil. Revista do Museu Paulista 4: 191300.

KARR, J.R. 1976. Seasonality, resource availability, and comunity diversity in tropical bird communities. American Naturalist 110: $973-994$

Liebezeit, J.R. \& T.L. George. 2002. Nest predators, nest site selection and nesting success of the Dusky Flycatcher in a managed ponderosa pine forest. Condor 104: 507-517.

LINDÉN, M. \& A.P. MøLLER. 1989. Cost of reproduction and covariation of life history traits in birds. TREE 4: 367-371.

LoPES, L.E. \& M.A. MARINI. 2005. Biologia reprodutiva de Suiriri affinis e S. islelorum (Aves: Tyrannidae) no cerrado do Brasil Central. Papéis Avulsos de Zoologia 45 (12): 127-141.

Machado, R.B.; L.M.S. Aguiar; A. Bianchi; R.L. Vianna; A.J.B. SanTOS; C.H. SAITO \& J.F. TIMmeRS. 1997. Áreas de risco no entorno de unidades de conservação: estudo de caso da Estação Ecológica de Águas Emendadas, Planaltina, DF, p. 64-73. In: J. Marinho-Filho, F. Rodrigues \& M. Guimarães (Eds). Vertebrados da Estação Ecológica de Águas Emendadas. História natural e ecologia em um fragmento de cerrado do Brasil Central. Brasília, SEMATEC, IEMA, IBAMA, 92p.

Marinho-FILho, J.; F. Rodrigues \& M. GuimarÃes. 1998. Vertebrados da Estação Ecológica de Águas Emendadas. História Natural e Ecologia em um fragmento de cerrado do Brasil Central. Brasília, SEMATEC, IEMA, IBAMA, 92p.

MARINI, M.Â. 1992. Notes on the breeding and reproductive biology of the Helmeted Manakin. Wilson Bulletin 104: 169-173.

Marını, M.Â. \& R.B. CavalCantı. 1990. Migrações de Elaenia albiceps chilensis e Elaenia chiriquensis arbivertex (Aves: Tyrannidae). Boletim do Museu Paraense Emílio Goeldi 6: $59-66$
MARTIN, T.E. 1987. Food as a limit on breeding birds: a life-history perspective. Annual Review of Ecology and Systematics 18: $453-487$.

MARTIN, T.E. 1993. Nest predation and nest sites. Bioscience 43: $523-532$.

MAYFIELD, H.F. 1975. Suggestions for calculating nest success. Wilson Bulletin 87: 456-467.

Mezquida, E.T. 2002. Nidificación de ocho especies de Tyrannidae en la Reserva de Ñacuñán, Mendoza, Argentina. Hornero 17: 31-40.

MonAgham, P. \& R.G. NAGeR. 1997. Why don't birdslay more eggs? Trends in Ecology and Evolution (TREE) 12: 270-274.

MuRPhy, M.T. 1986. Temporal components of reproductive variability in Eastern Kingbirds (Tyrannus tyrannus). Ecology 67: 1483-1492.

Naumburg, E.M.B. 1930. The birds of Matto Grosso, Brazil. A report on the birds secured by the Roosevelt-Rondon Expedition. Bulletin American Natural Museum of Natural History 60: 276-283.

Negret, A.; J. Taylor; R.C. Soares; R.B. Cavalcanti \& C. Johnson. 1984. Aves da região geopolítica do Distrito Federal. Brasília, SEMA, 21p.

Niceforo, H. \& A. Olivares. 1976. Addition to the Colombian avifauna: VI (Tyrannidae, Bombycilidae). Lozania 20: 1934.

Nimer, E. 1979. Climatologia do Brasil. Brasília, IBGE, 420p.

ONIKI, Y. \& E.O. WILLIS. 1983. Breeding records of birds from Manaus, Brazil; IV. Tyrannidae to Vireonidae. Revista Brasileira de Biologia 43: 45-54.

Partridge, W.H. 1964. Nota sobre la presencia de Elaenia chiriquensis e Tangara cayana em la avifauna argentina, con comentarios sobre Tangara arnaulti, Berlioz. Neotropica 10: 15-25.

Perrins, C.M. 1970. The timing of bird's breeding seasons. Ibis 112: 242-255.

Pichorim, M.; M.R. Bornschein \& B.L. Reinert. 1996. Aspectos da biologia reprodutiva de Knipolegus nigerrimus (Tyrannidae). Ararajuba 4: 29-31.

Pinto, O. 1944. Catálogo das aves do Brasil e lista dos exemplares existentes na coleção do Departamento de Zoologia. São Paulo, Departamento de Zoologia da Secretaria da Agricultura de São Paulo, 700p.

Ribeiro, J.F. \& B.M.T. Walter. 1998. Fitofisionomias do bioma Cerrado, p. 87-166. In: S. M. SANo \& S.P.D. AlmeIDA (Eds). Cerrado: ambiente e flora. Brasília, EMBRAPA, 556p.

RICKLEFS, R.E. 1990. The economy of nature: a textbook in basic ecology. New York, Chiron Press, 470p.

Ricklefs, R.E. 2000. Density dependence, evolutionary optimization, and the diversification of avian life histories. Condor 102: 9-22.

Ricklefs, R.E. \& G. BLoom. 1977. Components of avian breeding productivity. Auk 94: 86-96.

Ridgely, R.S. \& G. Tudor. 1994. The birds of South America, 
the Suboscine Passerines. Austin, University of Texas Press, $814 p$.

Robinson, T.R.; W.D. Robinson \& E.C. Edwards. 2000. Breeding ecology and nest-site selection of Song Wrens in central Panama. Auk 117: 345-354.

Roper, J.J. 2005. Try and try again: nest predation favors persistence in a neotropical bird. Ornitologia Neotropical 16: 253-262.

Saether, B.E. \& Ø. Bakke. 2000. Avian life history variation and contribution of demographic traits to the population growth rate. Ecology 81: 642-653.

Schauensee, R.M.D. 1982. A guide to the birds of South America. Philadelphia, Intercollegiate Press, 449p.

Sıck, H. 1997. Ornitologia Brasileira, uma introdução. Rio de Janeiro, Nova Fronteira, 912p.

SILVA, J.M.C. 1998. Integrating biogeography and conservation: an example with birds and plants of the Cerrado region, partell. Anais da Academia Brasileira de Ciências 70: 881888.

Silva, J.M.C. \& J.M. Bates. 2002. Biogeographic patterns and conservation in the South American Cerrado: A tropical savanna hotspot. BioScience 52: 225-233.

SILVA JR., M.C. \& J.M. FELFILI. 1996. A vegetação da Estação Ecológica de Águas Emendadas. Brasília, SEMATEC, 43p.

SKUTCH, A.F. 1945. Incubation and nestling periods of Central American birds. Auk 62: 8-37.

SkUTCH, A.F. 1960. Life histories of Central American birds II. Families Vireonidae, Sylviidae, Turdidae, Troglodytidae,
Paridae, Corvidae, Hirundinidae, and Tyrannidae. Pacific Coast Avifauna 34. Berkeley, Cooper Ornithological Society, 593p.

SKUTCH, A.F. 1966. A breeding bird census and nesting success in Central America. Ibis 108: 1-16.

SKUTCH, A.F. 1985. Clutch size, nesting success, and predation on nests of neotropical birds, reviewed. Ornithological Monographs 86: 575-593.

Stiles, F.G. \& A.F. SKUTCH. 1989. A guide to the birds of Costa Rica. Ithaca, Comstock Publishing, 511p.

Traylor JR, M.A. 1982. Notes on tyrant flycatchers. Fieldiana Zoology 13: 1-22.

Vasconcelos, M.F. \& J.A. Lombardi. 1996. Primeira descrição do ninho e do ovo de Polystictus superciliaris (Passeriforme: Tyrannidae) ocorrente na Serra do Curral, Minas Gerais. Ararajuba 4: 114-116.

Wetmore, A. 1972. The birds of Republic of Panama. Parte 3: Passeriformes. Dendrocolaptidae (Woodcreepers) to Oxyruncidae.(Sharpbills). Smithsonian Miscellaneous Collections 150: 1-631.

WilLiAms, G.C. 1966. Natural selection, the costs of reproduction, and a refinement of Lack's principle. American Naturalist 916 (100): 687-690.

YERKES, T. 1998. The influence of female age, body mass, and ambient conditions on Redhead incubation constancy. Condor 100: 62-68.

Yom-Tov, Y., M.I. Christie \& G.J. Iglesias. 1994. Clutch size in passerines of southern South America. Condor 96: 170-177.

Recebido em 24.I.2006; aceito em 01.III.2007.

Revista Brasileira de Zoologia 24 (1): 12-20, março 2007 